\title{
¿QUÉ ES UNA NORMA SOCIAL? Una discusión de tres aproximaciones analíticas
}

\author{
WHAT IS A SOCIAL NORM? \\ A discussion of three analytical approaches
}

\author{
JORDI TENA-SÁNCHEZ jordi.tena@uab.cat \\ Universitat Autònoma de Barcelona. España
}

ARIAdNA GüELL-SANS ariadnags@gmail.com

Institut de Seguretat Pública de Catalunya. España

\begin{abstract}
Resumen
En el presente trabajo se comparan las definiciones del concepto de "norma social" ofrecidas por la teoría de la elección racional estándar, Cristina Bicchieri y Jon Elster. Se trata de determinar en qué medida son o no asimilables. En la medida en que no lo son, se intenta establecer cuál de las aproximaciones captura mejor la naturaleza del fenómeno al que habitualmente denominamos norma social y cuál de ellas ofrece un concepto (o conceptos) más preciso y más fácil y fructiferamente utilizable en modelos explicativos. Se sostiene que la distinción trazada por Jon Elster entre normas morales, cuasi-morales y sociales tiene más éxito que las otras dos en dicho cometido. Pese a que el autor no prevé el uso de sus conceptos en modelos matemáticos formales con potencial predictivo, no hay nada inherente a los mismos que impida ese uso. Por otra parte, la precisión en la definición de los mismos los convierte en especialmente adecuados para ser utilizados en modelos de mecanismos que permitan ofrecer explicaciones causales parsimoniosas e informativas de los casos concretos.
\end{abstract}

\section{Palabras Clave}

Cristina Bicchieri, Jon Elster, Norma Moral, Norma Cuasi-Moral, Teoría de la Elección Racional.

\begin{abstract}
In this paper we compare the definitions of social norm proposed by standard Rational Choice Theory, Cristina Bicchieri and Jon Elster. We try to determine how similar or different they are. As long as they present some differences, we try to establish which captures better the very nature of the phenomena that we usually name "social norm" and which provides us with a concept (or concepts) more precise and productive and more easily useable in explicative models. In our opinion, Jon Elster's classification between moral, quasi-moral and social norms is the more successful in this respect. In spite of the fact that Jon Elster does not plan to use their concepts in formal mathematical models with predictive purposes, there is not anything inherent in them which prevents this use. On the other hand, the precision of these concepts makes them especially useful to be used in social mechanism models which let us propose causal, informative and parsimonious explanations of real concrete cases.
\end{abstract}

\section{KEYWORDS}

Cristina Bicchieri, Jon Elster, Moral Norm, Quasi-Moral Norm, Rational Choice Theory. 


\section{INTRODUCCIÓN ${ }^{1}$}

El de "norma social" es uno de los conceptos centrales tanto para la sociología como para las ciencias sociales y la filosofía en general. No obstante, pese a su recurrencia no existe aún hoy en día acuerdo sobre qué es exactamente una norma social. Contar con una definición precisa del concepto resulta, empero, fundamental para poder plantearse de qué forma motivan las normas la conducta, cómo emergen o de qué manera podemos incidir sobre ellas a través del diseño institucional, la educación o cualquier otro medio.

En ciencias naturales cuando se definen conceptos se establecen cortes en la realidad que permiten capturar una característica de la misma que está allí independientemente de nosotros y que adopta siempre la misma forma. En definitiva, se captura lo que en filosofía se denomina una "clase natural". En el mundo social, a diferencia del natural, parece que no sería posible hallar "clases naturales". La cuestión pasa a ser entonces, qué se le puede pedir a una "buena" definición en ciencias sociales. En dichas ciencias, un concepto está bien definido cuando es fructífero en términos explicativos, a saber, cuando es informativo y fácilmente operativizable, y permite ofrecer explicaciones causales parsimoniosas de los fenómenos sociales.

En los últimos años, las aproximaciones más relevantes al problema de las normas sociales han venido de la mano de los teóricos del ámbito de la teoría de la elección racional, siendo algunos de sus principales exponentes E. Ullmann-Margalit (1977) o J. Coleman (1991). No obstante, la publicación en 2006 del trabajo de C. Bicchieri The Grammar of Society volvió a revolucionar el debate. Por otra parte, Jon Elster ha propuesto en algunos de sus últimos trabajos $(2007 ; 2009)$ una distinción entre normas morales, cuasi-morales y sociales que pretende capturar mejor que la de Bicchieri el fenómeno al que habitualmente denominamos "norma". En el presente trabajo se discuten estos tres enfoques tratando de ver en qué medida son o no asimilables y, en la medida en que no lo son, cuál de ellos captura mejor el fenómeno y cuál de ellos ofrece un concepto (o conceptos) más preciso y más fácil y fructiferamente aplicable a modelos explicativos.

El trabajo se centra en la cuestión de qué debemos entender por una norma y de qué modo motivan la conducta los diferentes tipos de normas. Se deja de lado en estas páginas la pregunta, sin duda fundamental, de cómo emergen las normas. Asi mismo, tampoco se entra aquí a discutir fenómenos cercanos a las normas como pueden ser las reglas legales formales, las convenciones, las tradiciones o los hábitos y costumbres privados. Simplemente se señala que no se consideran normas sociales a ninguno de esos fenómenos.

\footnotetext{
${ }^{1}$ Este trabajo se ha beneficiado de los incisivos comentarios recibidos en el seminario Teoría Social Analítica -conste aquí nuestro agradecimiento a sus miembros-, así como del apoyo de un Proyecto $1+D+1$ con referencia CSO2009-09890 y de un proyecto CONSOLIDER-INGENIO con referencia CSD 2010-00034, ambos financiados por el Ministerio de Ciencia e Innovación (MICINN).
} 
En lo que sigue, se empezará presentando las distintas definiciones y tipologías de normas ofrecidas por los autores tratados en el trabajo. Posteriormente se intentará ver cuáles son las principales similitudes y diferencias entre esas aproximaciones poniendo el acento en algunas dimensiones clave del fenómeno. El trabajo finalizará con un apartado de conclusiones en el que tratará de dictaminarse cuál de las aproximaciones expuestas captura mejor el tipo de fenómenos al que se dirigen. En este sentido, se defenderá que es la clasificación de Jon Elster la que mejor alcanza su objetivo.

\section{HACIA UNA DEFINICIÓN DE NORMA SOCIAL}

\section{Las normas sociales en la Teoría de la Elección Racional estándar}

\section{La Teoría de la Elección Racional y las normas sociales}

Como es bien sabido, en su versión estándar (TER-e, en adelante), la teoría de la elección racional presupone que todas las acciones sociales se explican según las decisiones adoptadas por un individuo o conjunto de individuos en un determinado contexto. Se asume que dichos individuos tratan de maximizar su utilidad, a saber, ante un cierto conjunto de oportunidad, se supone que el individuo escogerá la opción que le reportará mayor beneficio (Aguiar y De Francisco 2009). En el modelo estándar, de modo particular, se asume que los individuos son egoístas ${ }^{2}$.

La TER-e desplazó el que había sido el centro de interés tradicional en los estudios sociológicos sobre normas sociales. Generalmente, los científicos sociales habían tomado las normas sociales como dadas y, a partir de ahí, se centraban en exclusiva en el impacto de estas sobre la conducta de los agentes. Los teóricos de la TER-e, en cambio, se preguntan principalmente acerca de cuál es el origen de dichas normas: estas pasan de ser concebidas como el explanans de la acción social a ser el explanandum del que hay que dar cuenta (Linares 2007).

\footnotetext{
${ }^{2}$ Para ser más precisos, la TER no necesita suponer que los agentes sean egoístas, sino únicamente "autointeresados". Es decir, si bien los individuos maximizan su función de utilidad, esta no tiene por qué referirse exclusivamente a su propio interés estrecho (Véase Sánchez-Cuenca 2007, para una discusión). No obstante, en el presente trabajo se deja de lado esta complicación y se asume que el supuesto de egoísmo universal es un elemento característico de la TER estándar. Esto es así porque, si bien este no es un requisito lógico de la teoría, en la práctica ese supuesto es el habitual entre la mayoría de los autores de la TER y en este sentido es uno de los elementos que mejor caracteriza a la versión estándar de la misma.
} 
¿Qué es una norma social?

Para los autores de esta corriente (Ulman-Margalit 1977, Coleman 1991) una norma social es, en primer lugar, un tipo de regularidad de conducta en el nivel macro (Linares 2007). La regularidad de conducta es, por tanto, un elemento constitutivo de las normas sociales. Si no hay regularidad de conducta no puede hablarse de la existencia de una norma. Obviamente, la existencia de regularidad de conducta no es suficiente para que se dé una norma, de lo contrario nos veríamos obligados a aceptar la conclusión contraintuitiva de tener que denominar norma a regularidades socialmente extendidas como comer cada mediodía o dormir cada noche. La norma social es primordialmente una característica de la estructura de interacción, no un estado mental interno de los individuos. Ésta se manifiesta en la forma de regularidad de conducta y de ejecución de sanciones (Linares 2007). Por tanto, ¿a qué se reduce ontológicamente, en el nivel micro, la propiedad macro en que consiste una norma? A acciones individuales similares, o sea, a una regularidad de conducta de un cierto tipo.

Por lo que se refiere a los restantes elementos que constituyen una norma social, pese a que puedan existir diferencias de tipo nominal entre los autores, de forma general, y más o menos explícita, en la literatura se hace referencia a "las expectativas o derechos de los actores, que implican necesariamente algún grado de consenso en el grupo; es decir, los miembros del grupo esperan los unos de los otros un determinado comportamiento" así como a "las condiciones de realización de la norma 0 , más precisamente, de la ejecución de sanciones. La existencia de un derecho a controlar el comportamiento de otro miembro del grupo se concreta en el derecho a ejecutar sanciones (positivas o negativas) sobre la conducta del mismo. Esto plantea el problema de que debe existir la posibilidad de sancionar para que una norma exista" (Linares 2007: 133).

\section{Las normas morales y sociales en la clasificación de C. Bicchieri}

\section{Una reconstrucción racional del seguimiento de normas sociales}

Cristina Bicchieri (2006) ofrece una "reconstrucción racional" del cumplimiento de normas sociales. La aproximación de Bicchieri entra dentro del ámbito de la TER ya que utiliza modelos matemáticos formales basados en las funciones de utilidad de los agentes. Aun así, la suya no es una aproximación ortodoxa. Bicchieri sostiene que cumplir normas es racional, no porque sea el resultado de un proceso de procesamiento perfecto de información y de maximización de utilidad esperada en términos egoístas, sino porque se puede dar cuenta del fenómeno a partir de las preferencias y las creencias de los agentes. Su tratamiento del problema difiere, por tanto, en este punto de la TER-e. La propuesta de Bicchieri comparte, empero, con la TER-e la ventaja de que resulta posible generar a partir de ella funciones de utilidad incorporables a modelos formales que ofrezcan explicaciones y predicciones informativas sobre la realidad. 


\section{Definición de norma social}

Según Bicchieri existen cuatro condiciones que son individualmente necesarias y conjuntamente suficientes para -y por tanto, constitutivas de- la existencia de una norma social. Éstas son: contingencia, expectativas empíricas, expectativas normativas y preferencias condicionales:

\section{Condiciones para la existencia de una norma social}

Sea $R$ una regla de conducta para situaciones del tipo $S$, donde $S$ puede representarse como un juego mixed-motive. Decimos que $R$ es una norma social en una población $P$ si existe un subgrupo suficientemente amplio $P S C \subseteq P$, de modo que para cada individuo $i \in P S$ :

1. Contingencia: $i$ sabe que la regla $R$ existe y que se aplica a situaciones del tipo $S$;

2. Preferencia Condicional: i prefiere cumplir con $R$ en situaciones del tipo $S$ a condición de que:

2a. Expectativas empíricas: $i$ cree que un subgrupo suficientemente amplio de $P$ cumple con $R$ en situaciones del tipo $S$;

2b. Expectativas normativas: $i$ cree que un subgrupo suficientemente amplio de $P$ espera que $i$ cumpla con $R$ en situaciones del tipo $S$; 0

$2 b$ '. Expectativas normativas con sanciones: $i$ cree que un subgrupo suficientemente amplio de $P$ espera que $i$ cumpla con $R$ en situaciones del tipo $S$, prefiere que $i$ cumpla y puede sancionar la conducta (2006:11, traducción de los autores) ${ }^{3}$.

En lo que sigue, siguiendo la numeración de la propia autora, nos referiremos al seguimiento de una norma (en la concepción de Bicchieri) sin necesidad de sanciones, como el caso $2 \mathrm{~b}$; mientras que el seguimiento de una norma por el efecto disuasorio de las sanciones se denominará caso $2 b^{\prime}$.

\section{Definición de norma moral}

Bicchieri distingue entre normas "morales" y "sociales". Las normas morales, a diferencia de las sociales, son incondicionales. Seguimos las normas morales independientemente de lo que hagan o piensen otros. Las seguimos porque contamos con buenas razones independientes para hacerlo. Las expectativas o las preferencias de los demás no proporcionan una buena razón para seguir una norma moral. Seguimos normas morales porque consideramos que son buenas en sí mismas, la razón para obedecer una norma de este tipo, por tanto, reside en el contenido de la norma misma.

\footnotetext{
${ }^{3}$ El subíndice "sc" de $P$ significa "seguidores condicionales" de la regla $R$. Se trata de la traducción de "Pcf", "conditional followers"
} 
Nótese como se pone el acento en los estados mentales de los agentes en la definición de la norma. Normas morales y sociales constituyen, ante todo, tipos de motivaciones diferentes. No hay nada en el mandato, en el contenido de la norma, que permita decir a priori si una determinada norma es moral o social. De esto se sigue que una norma puede ser moral para una población y social para otra. Más aún, puede ser que un determinado mandato motive a un agente en forma de norma social y a otro en forma de norma moral. Por ejemplo, usted puede pensar que reciclar las basuras es algo bueno y puede que, por tanto, se encuentre motivado o motivada para hacerlo. Pero puede que no esté usted dispuesta a ser la única que se esfuerce en reciclar la basura mientras que el resto de sus vecinos se abstienen de cooperar y convierten en inútil su esfuerzo. Si esto es así, puede que usted se encuentre motivada por una norma social. $\mathrm{Si}$, por el contrario, tiene usted una personalidad kantiana y sigue reciclando sus basuras independientemente de lo que hagan sus vecinos, puede que esté usted siguiendo una norma moral. En definitiva, por su naturaleza, las normas morales demandan un compromiso incondicional ${ }^{4}$.

\section{Las normas morales, cuasi-morales y sociales en la obra de Jon Elster}

Jon Elster marcó un antes y un después en cuanto al tratamiento de las normas sociales, al menos en el seno de la tradición analítica, con la publicación en 1989 [1991] de la primera edición de su The Cement of Society. En las dos décadas transcurridas desde entonces el noruego ha ido matizando y enriqueciendo su punto de vista sobre la cuestión. En los últimos tiempos $(2007 ; 2009)$ han visto la luz dos trabajos de Elster en los que retoma la cuestión de las normas ofreciendo una tipología en la que, entre otras cosas, distingue entre normas morales, cuasi-morales y sociales.

\section{Las normas morales, cuasi-morales y sociales}

Tanto las normas cuasi-morales como las sociales son condicionales. Las cuasi-morales son condicionales respecto a la conducta de otros agentes, las sociales lo son respecto a su presencia (Elster 2007:104). Un agente se halla motivado por una norma cuasimoral cuando está motivado para cumplir si, y sólo si, los demás también lo hacen. En cambio un agente se halla motivado por una norma social cuando se encuentra motivado para cumplir si, y sólo si, existen otros agentes que pueden observar o descubrir su incumplimiento. De forma más precisa, las normas sociales son:

\footnotetext{
${ }^{4}$ No obstante, Bicchieri concede que hasta las normas más claramente morales pueden ser condicionales en última instancia. Pese a que cualquiera de nosotros pueda considerar el respeto a la vida humana como una norma moral de primer orden, es dudoso sostener que no la violaríamos si nos hallásemos en un contexto de absoluta barbarie en el que nuestra propia supervivencia fuese en ello. No obstante, las normas morales ofrecen un grado de incondicionalidad respecto de las sociales que hace que aún merezca la pena (que sea explicativamente fructífero) mantener la distinción.
} 
[... mandatos no orientados al resultado para actuar o para abstenerse de hacerlo, sostenidos por las sanciones que otros aplican a los violadores de la norma. Estas sanciones, que van desde el castigo directo hasta el ostracismo, son a su vez sostenidas por el desprecio en el observador de la violación de la norma y la vergüenza en el destinatario de las sanciones. (2009:195) .

El contenido de algunas normas sociales es incondicional, son del tipo "haz X"; el de otras es condicional", "si otros hacen X, tú haz Y".

Normas cuasi-morales y sociales se pueden reforzar mutuamente. Si nosotros le vemos a usted arrojando un papel al suelo, es posible que no nos importe que usted nos vea a nosotros hacer lo mismo. Verle a usted transgrediendo una norma social nos permite transgredirla también a nosotros sin miedo a su reprobación. Por otra parte, verle transgredir una norma cuasi-moral hace que no nos sintamos obligados a tener que cumplirla nosotros. Si, en cambio, le vemos arrojar el papel a la papelera, la equidad y el miedo a la reprobación se pueden combinar para motivarnos a nosotros a tirar también nuestro papel a la papelera.

Las normas morales en cambio son incondicionales. Exactamente igual que en el caso de Bicchieri, no hay nada en el contenido de la norma que nos diga a priori de qué tipo es. Del mismo modo, una misma regularidad de conducta puede ser el resultado de la operación de normas diferentes e incluso de combinaciones de ellas. Recuperando un ejemplo anterior, puede que en nuestra ciudad la gente recicle sus basuras siguiendo una norma moral, mientras que en la suya lo hacen siguiendo una cuasi-moral, o tal vez una social. De modo más complejo, puede que algunos de los que reciclan la basura en nuestra ciudad lo hagan guiados por una norma moral, otros por una cuasi-moral y otros por una social. En general, resulta probable que la acción sea iniciada por los agentes motivados moralmente, que les sigan los motivados por normas cuasi-morales y que los últimos en incorporarse sean los que se encuentren motivados por normas sociales.

Las emociones tienen un papel crucial tanto en el cumplimiento de la norma como en la ejecución de las sanciones ${ }^{6}$ (Elster 1991; 2002; 2007; 2009). Las distintas emociones vinculadas a los distintos tipos de normas y el modo concreto en que estas operan son un elemento diferenciador fundamental entre normas morales y sociales así como entre normas morales y otro tipo de fenómenos cercanos como los hábitos o normas privadas. La figura 1 recoge las emociones relacionadas con las normas morales y sociales.

\footnotetext{
${ }^{5}$ Condicional o incondicional se refiere aquí al contenido de la norma, como acaba de decirse, las normas sociales son siempre condicionales a la presencia de un observador.

${ }^{6}$ Más aún, según Elster (2009), es probable que las emociones resulten fundamentales para entender por qué los seres humanos creamos normas de cualquiera de los tres tipos.
} 
Figura 1.
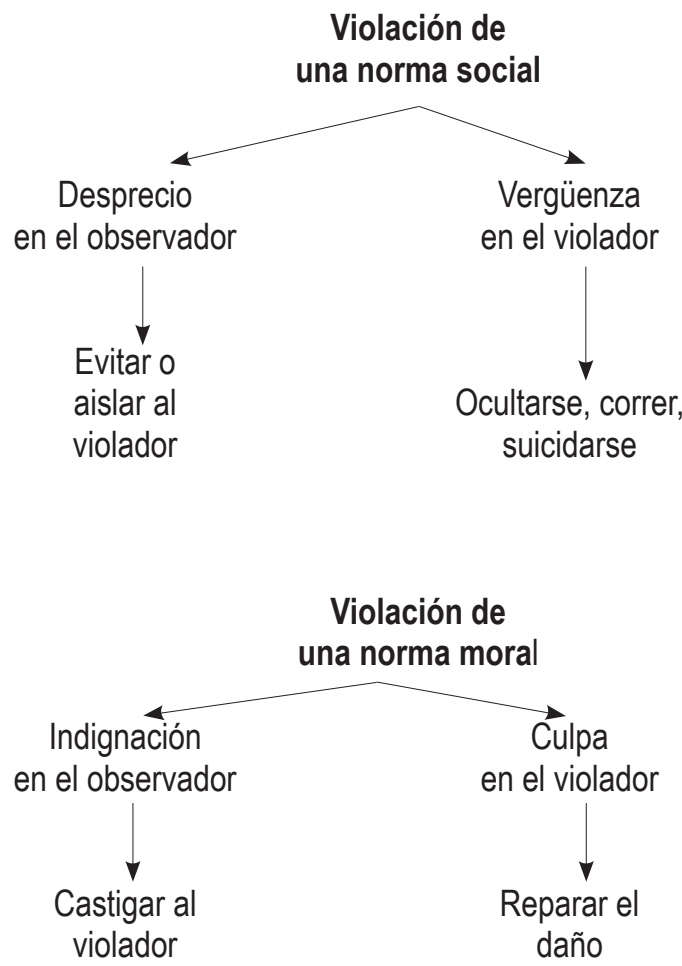

Tal y como puede observarse en la figura, la violación de una norma social causa desprecio en el observador y vergüenza en el violador. Por su parte, la violación de una norma moral genera indignación en el observador y culpa en el violador. El desprecio es producido por una creencia negativa sobre el carácter de alguien, concretamente por la creencia de que otro es inferior a uno mismo. La vergüenza es producida por una creencia negativa acerca del propio carácter. La indignación es generada por la creencia de que alguien ha realizado una mala acción. Finalmente, la culpa es generada por la creencia de que hemos realizado una mala acción ${ }^{7}$.

${ }^{7}$ Toda esta discusión se basa en Elster (2002; 2007; 2009). 
Las emociones dan lugar a "tendencias de acción". Una tendencia de acción puede ser entendida como una "preferencia temporal". Las dos partes del concepto resultan relevantes aquí. La segunda, el hecho de que la preferencia sea de carácter temporal, nos recuerda que las emociones pueden ser muy intensas pero también muy breves ${ }^{8}$. Pensar la tendencia de acción en términos de una "preferencia" temporal pone el acento en que las emociones no causan directamente la acción de manera mecánica o ciega. La influencia de las emociones se halla, por así decirlo, mediada por los deseos y las creencias de los individuos.

El desprecio que el observador de la violación de una norma social siente por el trasgresor genera como tendencia de acción evitar a este último. Por su parte, las tendencias de acción de la vergüenza consisten en tratar de ocultarse, correr 0 , en casos muy extremos, suicidarse. La indignación tiene como tendencia de acción tratar de infligir sufrimiento de sancionar, al trasgresor. Finalmente, la tendencia de acción de la culpa conduce a los individuos a confesar su falta y tratar de repararla y, en caso de que esto no sea posible, infligirse daño a ellos mismos.

La violación de normas morales y sociales no sólo produce emociones distintas sino que estas operan además a través de cadenas causales distintas. La violación de una norma moral producirá culpa en el violador independientemente de si es descubierto 0 no. En cambio, el violador de una norma social sólo siente vergüenza si es descubierto. Por otra parte, el desprecio, para ser efectivo, tiene que ser espontáneo. Si el desprecio no es espontáneo sino que es intencional, si se muestra desprecio con la voluntad deliberada de humillar al agente en cuestión, puede ser contraproducente y provocar indignación e incluso una reacción violenta en el agente en lugar de hacerle sentir vergüenza. Más aún, aunque el desprecio sea genuino, siempre puede ser percibido como intencional por el agente sancionado.

Elster no aclara cuáles son las emociones vinculadas a las normas cuasi-morales; aunque intuye que éstas son las mismas que soportan las morales, no tiene argumentos definitivos a favor de esta tesis. Tampoco aquí se dirá nada a este respecto.

Los límites entre los distintos tipos de normas y las emociones en que se apoyan no siempre resultan tan claros en los casos concretos. Pese a que las distintas normas hacen referencia a fenómenos analíticamente diferenciables, la frontera entre ellas es borrosa. Del mismo modo, o quizá como causa de lo anterior, las fronteras entre las distintas emociones vinculadas a las normas y entre éstas y las propias normas resultan también difusas. En un momento dado, por ejemplo, la violación de una norma social puede generar indignación, etc. (2002).

${ }^{8}$ No todas las emociones tienen por qué ser breves. Para una completa discusión, véase al citado Elster 


\section{ALGUNAS SIMILITUDES Y DISCREPANCIAS ENTRE LOS ENFOQUES}

\section{Sobre el tipo de situaciones en que se aplican las normas}

Para el conjunto de los autores de la TER-e citados, las normas existen "porque" promueven externalidades positivas o impiden que se produzcan negativas. Como se dijo, no se entrará aquí a discutir cuál sea el origen de las normas sociales, pero merece la pena destacar que este tipo de explicación presenta limitaciones importantes desde el momento en que resulta evidente que existen multitud de normas que no sirven para solucionar absolutamente nada o que incluso generan sufrimientos a todas luces innecesarios a las personas.

Por su parte, en su caracterización de las normas sociales, C. Bicchieri sostiene que una situación del tipo $S$, una situación regida por una determinada norma social, "se puede presentar como un mixed-motive game" (2006:11). Como es bien sabido, en teoría de juegos se denominan mixed-motive a aquellos juegos en los que los intereses de los agentes son parcialmente divergentes pero también parcialmente coincidentes. Dilemas como el del prisionero, el de la gallina o el del principal y agente serían algunos ejemplos célebres de este tipo de juegos. Sin embargo, cabe preguntarse acerca del porqué de tal requisito. Existen normas sociales que se aplican a situaciones que no presentan ese tipo de estructura y que, más aún, no sirven para solucionar ningún tipo de problema. Obviamente, una norma social, cuando existe, da lugar a un dilema en el sentido de que los que la consideren mala y traten de oponerse a ella se encontrarán ante un problema de acción colectiva. Pero claramente, eso no es lo que dice Bicchieri: ella sostiene que la norma "soluciona" un dilema "previamente existente" (2006:2-3 y 26-27). Pero, recogiendo un par de ejemplos de Elster, ¿cuál es el dilema previo al que dan solución normas como la que nos prohibe a nosotros ofrecerle a usted dinero para comprarle su plaza en la cola del autobús o las normas de venganza que rigen en determinadas sociedades?

En definitiva, la condición de que la situación $S$, necesariamente, "se puede presentar como un mixed-motive game", se antoja falsa. Más aún, da la impresión de que Bicchieri no ha logrado en este punto distanciarse del todo de ese aroma funcionalista que acompaña a las explicaciones acerca del origen de las normas sociales que suelen ofrecerse desde la TER-e.

\section{La regularidad de conducta en la definición de la norma}

\section{La regularidad de conducta en las normas sociales y cuasi-morales}

Una segunda cuestión importante se refiere al papel que juega la regularidad de conducta en lo que constituye una norma. Como se vio, que se dé dicha regularidad es un elemento imprescindible para que pueda hablarse de la existencia de una norma en el caso de la TER-e. No obstante, esto no es así para las normas sociales en el caso de Bicchieri, ni para las sociales y cuasi-morales en el caso de Jon Elster. Los argumentos ofrecidos por Bicchieri en este punto cierran la discusión a su favor y al de J. Elster. 
En el caso de Bicchieri, aunque generalmente es de esperar que la existencia de una norma social dé lugar a una regularidad de conducta, esto no tiene por qué ser así siempre. Por otra parte, aun cuando se da regularidad de conducta, ésta no constituye lo que la norma es, ya que la norma es constituida por los cuatro elementos mencionados más arriba: contingencia, expectativas empíricas, normativas y preferencias condicionales.

Una norma social existe si el conjunto de individuos que la alberga, el conjunto de seguidores condicionales (Psc) dentro de la población (P) es suficientemente numeroso ${ }^{9}$. Pero la norma social sólo se cumplirá de forma efectiva si el conjunto de individuos que efectivamente la siguen (Ps) es, nuevamente, suficientemente numeroso (Bicchieri 2006:12). Existen algunas situaciones en las que es posible que las condiciones enumeradas en la definición de la norma se cumplan, es decir, que la norma exista, pero que, sin embargo, ésta no se cumpla a saber, no se dé regularidad de conducta alguna.

En primer lugar, podemos hallarnos ante un casode "ignoranciapluralista"10 combinado con una norma el cumplimiento de la cual no puede ser observado directamente (2006:12). Por ejemplo, imaginemos una comunidad en la que existe una norma en contra de las relaciones sexuales prematrimoniales. La norma existe, ya que se cumple que los individuos saben que existe $R$ ("abstenerse de mantener relaciones sexuales") y que se aplica a $S$ (relaciones entre individuos solteros). En una situación así el individuo i cree que los demás están cumpliendo, que esperan que él haga lo mismo y que van a sancionarlo duramente si lo descubren incumpliendo la norma. Dadas todas estas condiciones, el individuo $i$ ( $y$ todos los demás) prefiere cumplir. Pero, evidentemente, es muy difícil determinar si la conducta en cuestión se cumple o no. De hecho, en este tipo de normas que regulan la conducta privada, lo que se observa son las consecuencias del cumplimiento, no el cumplimiento en sí. En este caso observamos la ausencia de embarazos entre las mujeres solteras, de lo que es posible inferir, erróneamente o no, que la norma se cumple. No obstante, en este y en otros muchos casos, es posible que observemos las consecuencias del cumplimiento sin que este se produzca realmente. En este ejemplo, puede que las parejas solteras utilicen métodos anticonceptivos de algún tipo. De este modo, finalmente puede que prácticamente nadie cumpla con la norma pero que esta continúe existiendo y siendo efectiva. Pese a que nadie cumple, puede que todos piensen que los demás cumplen y que esperan que ellos lo hagan. Es más, si alguien es descubierto, puede que sea incluso más duramente sancionado por aquellos que no siguen la norma que por aquellos que sí lo hacen. Al fin y al cabo, mostrar conformidad con la norma explícitamente es un buen medio para disipar dudas sobre el propio cumplimiento.

\footnotetext{
${ }^{9}$ Lo que cuente como "suficientemente numeroso" es una cuestión empírica que deberá dictaminarse en cada caso concreto.

${ }^{10} \mathrm{La}$ "ignorancia pluralista" (pluralistic ignorance) se da cuando la gente cree erróneamente que sus creencias y deseos son diferentes de los de sus pares, cosa que les lleva a ocultar sus puntos de vista y/o a comportarse del modo requerido por lo que ellos creen que es el consenso. Este fenómeno tiene numerosas consecuencias, de forma destacada, la mencionada perpetuación de normas impopulares. Véase el seminal trabajo de Miller y Prentice (1994) para una completa discusión.
} 
En segundo lugar, una norma puede existir y no ser seguida, debido a que los seguidores condicionales de la misma (Psc) se ven siempre enfrentados a un juego bayesiano que no tiene por qué concluir necesariamente siempre con el cumplimiento. Veamos esto con mayor detalle.

Según Bicchieri, una norma da solución a un mixed-motive game. Recordemos que un mixed-motive game es un tipo de juego de estrategia en el que los intereses de los jugadores resultan parcialmente divergentes pero también parcialmente coincidentes. Que las normas sociales den solución a un juego de este estilo significa que transforman un dilema original de estas características en un juego de coordinación y ayudan a los jugadores a seleccionar el equilibrio óptimo en este último.

Imaginemos que los agentes se encuentran en una situación $S$ que puede ser modelizada como un problema de bienes públicos, más concretamente como un dilema del prisionero, y que no existe ninguna norma social. En una situación así, el orden de preferencias de cada agente será el que se muestra en la figura 2.

Figura 2.

\begin{tabular}{|c|c|c|}
\hline & \multicolumn{2}{|c|}{ Others } \\
\hline & C & D \\
\hline C & $\mathrm{S}, \mathrm{S}$ & W, $\mathrm{B}$ \\
\hline$D$ & $\mathrm{~B}, \mathrm{~W}$ & $\begin{array}{l}\mathrm{T}, \mathrm{T} \\
\end{array}$ \\
\hline
\end{tabular}

Fuente: Bicchieri (2006:26).

Las letras que se encuentran en el interior de la tabla corresponden al inglés: $B$ : best, $\mathrm{S}$ : second best, T: third best y W: worst. Vemos como el orden de preferencias corresponde con el del mencionado dilema del prisionero. Si una norma que prescribe la mutua cooperación entra en funcionamiento y, por tanto, se cumplen las condiciones anteriores, las preferencias de los agentes se transformarán de tal modo que el mixed-motive game previo se transformará en un juego de coordinación de este estilo.

Figura 3.

Others

\begin{tabular}{|c|c|c|c|}
\hline \multirow{3}{*}{ Self } & & C & D \\
\hline & $C$ & $B, B$ & $\mathrm{~W}, \mathrm{~T}$ \\
\hline & D & T,W & $S, S$ \\
\hline
\end{tabular}

Fuente: Bicchieri (2006:26). 
En este nuevo juego podemos observar la existencia de dos equilibrios de Nas$\mathrm{h}^{11}$ con estrategias puras $(C, C$ y $D, D)$, con la característica de que el primero de ellos es óptimo de Pareto respecto del segundo que es subóptimo ${ }^{12}$. Ahora todos los agentes tendrán interés en coordinarse en torno al equilibrio óptimo pero puede que no lo logren por un problema de confianza.

Es importante recordar en este punto que para que una norma exista no es necesario que todos y cada uno de los individuos en $P$ se encuentren motivados por la misma. Siempre habrá personas que no se hallen motivadas para cumplir y que, por tanto, continúen jugando el dilema del prisionero original. Veamos qué puede llegar a implicar lo anterior a partir de un ejemplo con sólo dos jugadores. Siempre que un Psc se encuentre frente a un agente desconocido sobre el que no tiene información, deberá contemplar la posibilidad de hallarse ante un free-rider que tratará de explotarlo. Por tanto, los Psc se ven habitualmente enfrentados a juegos bayesianos, a situaciones de "riesgo", en las que sólo pueden saber que, o bien están jugando un mixed-motive game con probabilidad $p$, o bien están jugando un juego de coordinación con probabilidad 1-p. En estas circunstancias, resulta plausible suponer que en ocasiones dos Pcf se encuentren en una situación $S$ y que, pese a hallarse motivados para cumplir la norma existente, ninguno de los dos lo haga por un problema de confianza.

En la clasificación de Jon Elster, la existencia de regularidad de conducta no es condición necesaria ni suficiente para las normas cuasi-morales ni para las sociales. En el caso de las primeras vuelve a plantearse la cuestión del juego bayesiano. Recuérdese que, como en el caso $2 b$ de Bicchieri (la norma se sigue sin necesidad de sanciones), un agente motivado por una norma cuasi-moral sólo cumplirá si los demás también lo hacen. Por su parte, el ejemplo de la ignorancia pluralista afectaría a las normas sociales de Elster. Recuérdese ahora que, como en el caso 2b' de Bicchieri, un agente motivado por una norma de este tipo únicamente cumplirá si piensa que su incumplimiento puede ser descubierto.

\section{La regularidad de conducta en las normas morales}

Tanto para Bicchieri como para Jon Elster, las normas morales son incondicionales, por tanto se siguen siempre, independientemente de las consecuencias que eso tenga para el agente. De este modo, de la postura de los autores se deriva que la existencia de una norma moral necesariamente implica regularidad de conducta en el nivel macro' ${ }^{13}$.

\footnotetext{
${ }^{11}$ Como es bien sabido, el equilibrio de Nash es un conjunto de estrategias que tienen la propiedad de que ningún jugador puede aumentar sus pagos cambiando de estrategia unilateralmente. Dicho de otro modo, el equilibrio de Nash representa la solución de un juego de estrategia.

${ }^{12}$ Como también es bien sabido, un equilibrio es óptimo paretianamente cuando no existe otro resultado que aumente los pagos de al menos uno de los jugadores sin reducir los de ninguno. A la inversa, un equilibrio es subóptimo cuando sí que existe esa posibilidad.

${ }^{13}$ Podría argumentarse que esto no sería así, por ejemplo, en un caso de debilidad de la voluntad
} 
Reconocer este hecho nos enfrenta a una controversia de tipo ontológico. ¿Qué papel debe desempeñar la regularidad de conducta en la definición de la norma? ¿A qué es reducible en el nivel micro la norma? Para responder a estas preguntas resulta necesario tener presente que la principal virtud que podemos demandarle a la definición de un concepto en ciencias sociales es que sea fructífera en términos explicativos. Con esta precaución en mente, se sostendrá que la regularidad de conducta no forma parte de la definición de lo que es una norma moral y que ésta, al igual que la social o la cuasimoral, es reducible ontológicamente en el nivel micro a estados mentales de los individuos. Es cierto que podría decirse que la norma moral es una regularidad de conducta y que, por tanto, debe poder ser reducible a acciones individuales y tendríamos una definición aparentemente diferente de la que acabamos de ofrecer. Pero esto parece llevarnos a un juego de palabras, al fin y al cabo, las acciones son a su vez explicables a partir de estados mentales individuales. Poner el foco directamente sobre los estados mentales permite destacar que las normas morales son motivaciones para la conducta igual que lo son la racionalidad, las emociones o las demás normas y se diferencian de todas estas otras motivaciones por la forma que adoptan, por el modo en que operan, etc. En cambio, fijarse en primer término en la regularidad de conducta desvía la atención de cuestiones importantes ya que, como se dijo, una misma regularidad puede ser el fruto de muchos tipos distintos de motivación. Desde este punto de vista, es preferible contemplar la regularidad de conducta como un subproducto a nivel agregado de la existencia de una norma.

\section{Las normas como fuentes de motivación para la conducta de los individuos}

\section{Sobre la reducción de las normas sociales a racionalidad instrumental en la TER-e}

Tal y como se mencionó anteriormente, los autores de la TER-e desplazan el centro de atención desde la tradicional preocupación por el modo en que las normas influyen sobre la conducta hasta los mecanismos a través de los cuales éstas emergen. Eso hace que, en general, no se preste especial atención al problema que nos ocupa en este apartado. En palabras del propio Coleman "Tengo poco que decir sobre el cumplimiento de normas, el cumplimiento o el incumplimiento son simplemente el resultado de la aplicación del principio de maxinización de utilidad bajo diferentes constricciones Tengo poco que decir sobre el cumplimiento de normas, el cumplimiento o el incumplimiento son simplemente el resultado de la aplicación del principio de maxinización de utilidad bajo diferentes constricciones" (Traducción de los autores 1991:286).

socialmente extendida. Este contraejemplo vendría a favor de la tesis de fondo que se está defendiendo en estas páginas según la cual la regularidad de conducta no es constitutiva de las normas. No obstante, dado lo extraño del caso, se opta por dejarlo aquí de lado y asumir que la existencia de una norma moral implica necesariamente que se produzca una regularidad de conducta. 
Generalmente, para estos autores la norma no tiene fuerza motivadora por sí misma, independientemente de las sanciones que la acompañan. La norma es reducible a racionalidad instrumental. Según Linares, desde esta perspectiva, "la acción no se presupone guiada por una motivación vinculada a un entendimiento compartido del "deber ser" sino por algún tipo de cálculo de coste y beneficio" (2007:132). De este modo, la norma prevé sanciones positivas para los cumplidores y/o negativas para los incumplidores. El agente cumplirá con la norma si, y solo si, los beneficios que anticipa por cumplir superan a los del incumplimiento. Nótese que lo dicho no vale solo para el cumplimiento de las normas sociales de primer orden, sino también para la aplicación de sanciones. El agente solamente sancionará al incumplidor de una norma si existe una "metanorma" que así lo prescribe.

La postura de algunos autores, principalmente Coleman (1991), resulta algo más compleja en este punto. Pese a que ciertamente es para él una cuestión menor y secundaria, Coleman contempla la posibilidad de que los agentes interioricen determinadas normas sociales. Nuevamente, el autor reduce este hecho a racionalidad instrumental y sostiene que es posible que un agente termine por interiorizar una norma cuando, o bien es racional para él hacerlo 0 , tal vez más frecuentemente, es racional para un segundo agente tratar de que el primero interiorice la norma. En el ejemplo más obvio, los padres tratan de que sus hijos interioricen aquellas normas sociales que les permitirán afrontar la vida con mayores garantías de éxito. Cuando un agente interioriza una norma un sistema de sanciones "internas" se añade al sistema de sanciones "externas" previamente existente. A saber, una vez que un agente tiene una norma interiorizada, si opta por violarla, no sólo tendrá que hacer frente al reproche de los demás agentes si es descubierto, sino que también es probable que sienta determinadas emociones desagradables como la culpa. De este modo Coleman pretende explicar por qué en numerosas ocasiones los agentes cumplen con las normas sociales (o sancionan a los incumplidores) incluso en aquellos casos en que saben que no son observados y que su incumplimiento no sería descubierto.

A partir de ahí, la lógica es la misma que en el caso anterior en que sólo hay sanciones externas: el agente cumplirá si, y solo si, los beneficios anticipados de tal conducta exceden a los costes (ahora internos y externos) de los castigos derivados del incumplimiento. De modo general, la postura de la TER-e en este punto presenta muchos aspectos problemáticos. Algunos son de orden lógico mientras que otros son de tipo empírico, es decir, el concepto captura mal el tipo de motivación que impulsa a los agentes a actuar en muchos casos reales relevantes.

Es falso que las normas sociales sean reducibles a racionalidad instrumental, o, más generalmente, a intereses de algún tipo (Elster 1991). Pese a que resulta obvio que todo el mundo con mayor o menor frecuencia cumple con lo prescrito o lo proscrito por determinadas normas de manera instrumental, simplemente para lograr las recompensas 0 evitar las sanciones derivadas del cumplimiento o del incumplimiento, argumentar que eso es todo lo que sucede constituye una tesis manifiestamente falsa. Las normas no necesitan de sanciones externas para ser efectivas. Violar normas nos produce emo- 
ciones negativas que pueden disuadirnos de hacerlo aun en aquellas circunstancias en que no existe riesgo de ser descubiertos (Elster 1991; 2002). Un problema adicional del argumento es el de las "metanormas". ¿Por qué debería un agente racional que observa la violación de una norma aplicar una sanción al incumplidor? Este problema se discutirá de forma específica más adelante.

Antes se dijo que Coleman contempla la posibilidad de que los agentes interioricen la norma y que, en cierta manera, estas motiven la conducta a través de sanciones internas que se suman a las externas. Pero, tal y como en su momento mostrara Jon Elster (2002), existe un error lógico en el argumento de Coleman. No tiene sentido tratar emociones como la culpa o la vergüenza como un simple coste en una función de utilidad.

Imaginemos que alguno de nosotros se encuentra en la biblioteca consultando una nueva edición revisada del Foundations of Social Theory y que, en un momento dado, se percata de que el bibliotecario está despistado y que dispone de la oportunidad de robar el libro sin ser descubierto. Si esa persona es racional y si la culpa no es más que un mero coste, no dudará en robar el libro siempre que el valor que le atribuye a este supere la intensidad de la culpa que anticipa que sufrirá. El argumento es interesante porque pone el acento en la cuestión de la existencia de trade-offs entre racionalidad, emociones y normas pero, formulado de este modo, simplemente no tiene sentido. Tal y como sostiene Elster en un ejemplo ya clásico, siguiendo esa misma lógica, si existiese una pastilla a un precio módico en el mercado que permitiese a dicha persona evitar sentir culpa por violar normas (por robar el libro, por ejemplo) y si ella contase la culpa como un mero coste más o menos elevado, debería comprarla. Pero, conceptualmente esto nos conduce a una paradoja. Ninguna persona susceptible de ser disuadida por la culpa compraría la píldora ya que ello le produciría una culpa tal vez aún mayor. Por otra parte, alguien que estuviese dispuesto a comprarla, no necesitaría hacerlo puesto que ya se trataría de un sinvergüenza sin escrúpulos. En este sentido, no existe ninguna diferencia entre querer ser inmoral y serlo realmente.

En definitiva, las normas constituyen fuentes de motivación para la conducta distintas del egoísmo y no son reducibles a aquél en ningún caso.

\section{Las normas morales, cuasi-morales y sociales}

El tratamiento de la cuestión llevado a cabo por $\mathrm{C}$. Bicchieri y Jon Elster resulta más adecuado. Pese a que la postura de ambos autores resulta relativamente similar en este punto, existen discrepancias concretas que resulta relevante discutir y que son decisivas de cara a determinar cuál de las dos aproximaciones captura mejor el fenómeno al que se dirigen.

Para ambos autores, los individuos siguen las normas morales independientemente de las sanciones que éstas puedan llevar asociadas. Dichas normas motivan la conducta por sí mismas, por así decirlo, y no son reducibles en ningún caso a interés egoísta. Este punto es lo suficientemente claro como para no tener que discutirlo con mayor detalle. 
Por lo que se refiere al tratamiento de las normas sociales efectuado por $C$. Bicchieri, la distinción entre las condiciones 2 b y 2 b' resulta crucial aquí. Con ella se trata de capturar el hecho de que diferentes individuos pueden tener diferentes razones para "preferir" cumplir una norma. La condición $2 b$ se refiere a una expectativa recíproca: por una parte usted cree que los demás harán $X$, por otra parte usted cree que los demás creen que usted hará $X$. Dicha creencia puede ser de naturaleza empírica. Si los demás le han observado a usted haciendo $X$ en otras ocasiones resultará razonable para ellos pensar que usted también hará $X$ en esta ocasión. Pero la expectativa puede ser también normativa, puede que los demás piensen que usted "debe" hacer X. Para algunos individuos, en algunas ocasiones la satisfacción de esta condición $2 b$ (junto a las tres anteriores, claro está) será suficiente para que prefieran cumplir la norma. La existencia de sanciones será irrelevante en estos casos, no es el miedo a los castigos derivados del incumplimiento ni a las recompensas derivadas del cumplimiento lo que motiva al agente para cumplir. El reconocimiento de la legitimidad de las expectativas de los demás, la conformidad con la norma, es suficiente. En este caso la norma tiene fuerza motivadora por sí misma, por así decirlo.

En el caso de la clasificación de Jon Elster, el concepto de norma cuasi-moral trata de capturar exactamente el mismo fenómeno que el de norma social en el caso $2 b$ de Bicchieri. También en este caso, el cumplimiento no depende de la existencia de sanciones sino de la conducta de los demás (o de las expectativas sobre la misma).

La cuestión es más compleja en el caso de las normas sociales de Jon Elster y en el caso 2b' de Bicchieri. Con la condición 2b' Bicchieri trata de capturar el hecho obvio de que siempre existen agentes que no se hallan motivados por la norma y que no la cumplirán a menos que existan sanciones. En este punto cabe preguntarse si las condiciones $2 b$ y $2 b$ ' hacen referencia a dos formas - $a$ dos tipos de razones- diferentes de seguir las mismas normas o si se trata más bien de fenómenos diferentes. Es preciso recordar que las normas son fuentes de motivación para la conducta, reducibles a estados mentales. En este sentido, el mandato "haz X" ("recicla tu basura", por ejemplo) no es en sí mismo ni moral ni social. Como va dicho, puede, por ejemplo, que en una sociedad se trate de una norma moral y que en otra sea social, mientras que en una tercera simplemente no constituya una norma de ningún tipo. Más aún, puede que en una misma sociedad para un número suficiente de individuos "haz X" ("recicla tu basura") sea una norma moral, que para otros sea una norma social y que para otros no sea una norma de ningún tipo. Pero, nuevamente, si una norma es una fuente de motivación, ¿por qué sostener que en el caso $2 b$ y $2 b$ ' estamos ante la misma norma que motiva la conducta de formas diferentes? ¿Por qué no explorar la posibilidad de que se trate del mismo mandato, de la misma $R$, que da lugar a dos normas distintas que motivan de forma distinta y que tienen consecuencias distintas sobre la conducta? En este sentido, la definición de norma social de C. Bicchieri parece enfrentarse a algunas dificultades importantes en este punto. Según la autora, cuando se cumplen las condiciones de contingencia, preferencia condicional, expectativas empíricas y expectativas normativas (2b); la norma social ya existe. Si esto es así, cabe preguntarse qué añade la condición 
$2 b$ ' a la definición. Dicho de otra manera, la única diferencia entre las condiciones $2 b$ y $2 b^{\prime}$ es que la segunda incorpora sanciones. Por tanto, si cuando se cumple la condición $2 b$ ya existe la norma, ¿qué aportan las sanciones a la definición de la misma?

La distinción trazada por Elster entre normas cuasi-morales y sociales captura mejor el fenómeno que la distinción de $C$. Bicchieri entre expectativas normativas de tipo $2 b$ y $2 b$ ' (sin sanciones o con sanciones). La distinción de Elster ilumina mejor el hecho de que no se trata de dos formas alternativas de seguir la misma norma sino que se trata de fuentes de motivación diferentes, que influyen sobre la conducta de forma diferente y que se apoyan sobre emociones diferentes conectadas a las violaciones de las normas a través de cadenas causales también diferentes. La distinción de Elster en definitiva, y esta es la tesis más importante que se pretende defender en este trabajo, es la que mejor captura el fenómeno al que se dirige y se acerca más al ideal de la "clase natural".

\section{El problema de las metanormas}

Solucionar el dilema de las metanormas supone uno de los grandes retos a los que se enfrenta la aproximación a las normas sociales de los autores de la TER-e. Aplicar sanciones al incumplidor de una norma tiene exactamente la misma estructura de bien público que el cumplir con la norma en cuestión. Si nosotros observamos a un agente violando una norma, ¿por qué deberíamos sancionarlo? Aplicar sanciones siempre conlleva costes para el sancionador (Elster 1991; 2002; 2007; 2009), por tanto, si el agente que observa la violación es racional deberá abstenerse de sancionar y esperar a que lo haga otro. Los autores de la TER-e tratan de resolver el dilema argumentando que para que una norma social sea aplicada será necesario que exista una metanorma que obligue a quien observa la violación de una norma a sancionar si no quiere ser sancionado. De este modo, ya tenemos una respuesta para la pregunta de por qué debería el observador de un incumplimiento sancionar al incumplidor. La respuesta es que el observador sancionará para evitar a su vez ser sancionado. Sin embargo, salta a la vista que esta explicación tiene todo el aspecto de ser una pseudo-explicación que inmediatamente nos arroja a una regresión al infinito. Ciertamente algunos autores han logrado elaborar complejos modelos formales en los que muestran que si se dan ciertas condiciones (frecuentemente muy exigentes), la norma puede llegar a convertirse en un equilibrio estable. No obstante, tal y como reconoce Linares (2007), el problema de las metanormas dista mucho de estar resuelto.

Es evidente que el mecanismo ofrecido desde la TER-e opera en algunos casos y que hay gente que en ocasiones sanciona sólo para evitar ser sancionada. No obstante, pretender que eso es el único o el más importante factor que explica la aplicación de sanciones resulta poco plausible empíricamente y poco parsimonioso teóricamente. Es poco plausible empíricamente porque el mecanismo se vuelve más y más inverosímil conforme nos alejamos de la violación original. Resulta difícil pensar que alguien vaya a sancionar a quien se abstuvo de sancionar, a quien se abstuvo de sancionar, a quien se abstuvo de sancionar, a quien se abstuvo de sancionar al incumplidor de una norma. Es 
poco parsimonioso porque es extremadamente complejo y porque contamos con explicaciones más sencillas y mejor asentadas sobre la evidencia empírica que apuntan a las emociones como el mecanismo que da cuenta de la aplicación de sanciones. Nuevamente, es Jon Elster quien mejor resuelve esta cuestión. Las emociones son el "motor inmóvil" que da solución al problema de las metanormas (1991; 2002; 2007; 2009). Sancionamos al violador de una norma porque existe una fuerza psíquica muy poderosa que nos impulsa a hacerlo y que puede llegar a desbordar, y frecuentemente lo hace, los cálculos de costes y beneficios que podamos llegar a hacer. La indignación o el desprecio que nos produce ver a alguien violando una norma es la motivación que nos empuja a sancionar aunque, claro está, existen trade offs entre esa emoción y la racionalidad que nos pueden llevar a no sancionar si los costes que anticipamos son muy elevados.

Otra cuestión estrechamente relacionada con aplicar sanciones a los violadores de normas es la de los costes materiales. Ser sancionado por violar una norma puede tener importantes costes materiales para el destinatario de la sanción. Puede, por ejemplo, suponerle costes en términos de capital social o directamente en términos de capital físico si los demás se niegan a establecer relaciones económicas con él. La amenaza de sanciones de este tipo también constituye, obviamente, un incentivo para cumplir con lo prescrito por las normas de uno u otro tipo. Sin embargo, la cara más interesante de este fenómeno se encuentra en el lado contrario, en las motivaciones que impulsan al sancionador a incurrir en costes materiales al ejecutar la sanción. La explicación vuelve a encontrarse en la indignación o el desprecio que nos produce observar a alguien violando normas que nosotros compartimos. En este sentido, la sanción material efectiva parece tener más importancia en tanto que vehículo transmisor de la emoción de la que tiene por sí misma. Es decir, existen dos maneras de entender las emociones (desprecio o indignación) que acompañan a la sanción. En primer lugar pueden entenderse como constitutivas de la sanción (inmaterial), en segundo lugar pueden entenderse también como causa de la sanción (material). Aunque las dos dimensiones se dan en la realidad, la primera parece ser más importante. En términos de Jon Elster:

El aspecto material de la sanción que importa es cuánto le cuesta al sancionador penalizar al destinatario y no cuánto le cuesta al destinatario ser penalizado. Cuanto más me cueste negarme a tratar contigo, con más fuerza sentirás el desprecio que hay detrás de mi negativa y más acuciante será tu vergüenza. [...] Aunque los elevados costes para el sancionador suelen ir acompañados de costes elevados para el destinatario, [...], ese no tiene por qué ser el caso. E incluso cuando es el caso, lo que afirmo es que los costes para el sancionador es lo que convierten la sanción en realmente dolorosa para el destinatario. (2002:180-181).

La dimensión constitutiva o no de la emoción respecto de la sanción presenta otro aspecto interesante. La indignación es una emoción más intensa que el desprecio y suele conducir a la sanción efectiva del violador con mayor frecuencia que este último. Cuando observamos a alguien violando una norma social y sentimos desprecio, es poco probable que recriminemos directamente la conducta en cuestión. De hecho, la tenden- 
cia de acción del desprecio, como se vio, consiste en la evitación del despreciado. Dicha evitación, o el simple hecho de que se nos note que sentimos desprecio, constituye ya una fuerte sanción. El desprecio es, en una importante medida, actitudinal, por así decirlo. En cambio, la indignación suele conducir con mayor frecuencia a la sanción efectiva del violador. Como se vio, la tendencia de acción de la indignación consiste en tratar de dañar (sancionar explícitamente) al violador.

\section{El no-consecuencialismo de la conducta guiada por normas}

Otro tema de crucial importancia que separa a los autores tratados en el presente trabajo es el de si la conducta guiada por normas es o no de tipo consecuencialista. La respuesta es sí para los autores de la TER-e. Este hecho no resulta sorprendente, al fin y al cabo la conducta guiada por normas es reducible a racionalidad instrumental. La respuesta es no para Jon Elster, pues según él, uno de los elementos característicos de la motivación para seguir normas es el no-consecuencialismo. Ese no consecuencialismo constituye precisamente uno de los principales ejes de distinción, si no el principal, entre normas y racionalidad. En lo que se refiere a $\mathrm{C}$. Bicchieri, según la interpretación efectuada en estas páginas, el seguimiento de las normas morales y de las sociales en el caso $2 b$ son de tipo no consecuencialista, mientras que en el caso $2 b$ ' serían de tipo consecuencialista. Los argumentos ofrecidos por J. Elster vuelven a ser definitivos en este punto y ofrecen una razón más para preferir su clasificación a la de los autores de la TER-e y a la de Bicchieri.

En primer lugar, que las normas morales no son consecuencialistas es una conclusión lógica de su naturaleza. Las normas morales deben cumplirse siempre, independientemente de lo que hagan los demás o de las consecuencias que tal conducta pueda llegar a tener.

Las normas cuasi-morales de Jon Elster tampoco son consecuencialistas. Esto se deriva del hecho de que, más que estar orientadas hacia el futuro, lo están hacia el pasado. La razón para cumplir con una norma cuasi-moral es que otros lo han hecho y eso nos motiva para actuar independientemente de las consecuencias que pueda tener nuestra acción.

El seguimiento de normas sociales en el caso $2 \mathrm{~b}$ de $\mathrm{C}$. Bicchieri es también de tipo no-consecuencialista. El no-consecuencialismo es lo que distingue a una simple preferencia por un determinado producto de la acción de una preferencia condicional por seguir una norma (Bicchieri 2006:57-58). Recogiendo el mismo ejemplo puesto por la autora, no es lo mismo tener una preferencia por la equidad que tener una preferencia condicional para seguir una norma de equidad. La primera es una motivación orientada a futuro, lo que interesa es, estrictamente, el producto de la acción. La segunda es una preferencia orientada al proceso, lo que preferimos (condicionalmente) es actuar de forma justa, independientemente de las consecuencias. Finalmente, es necesario abordar la cuestión del consecuencialismo del seguimiento de normas sociales en el caso 2b' 
de Bicchieri y de normas sociales en la clasificación de Jon Elster. Parece obvio que el cumplimiento de una norma en el caso 2b' de Bicchieri se entiende de modo consecuencialista. Esto no es así para el caso de las normas sociales de Jon Elster. Una vez más, es el noruego quien mejor captura la naturaleza del fenómeno.

Las normas sociales se sostienen, según Elster, por las sanciones aplicadas a los violadores. Este hecho parecería conducirnos a la conclusión de que cumplir una norma social es un tipo de conducta consecuencialista e, incluso, reducible a racionalidad instrumental, pero en realidad esto no es así. Diferentes argumentos pueden ser ofrecidos aquí. En primer lugar, como se dijo, el sancionador de la norma actúa de forma incondicional. En segundo lugar, es necesario tener en cuenta la operación del cold to hot empathy gap y del hot to cold empathy gap (Elster 2009b). Tal y como ha sido mostrado por la psicología, cuando los individuos se encuentran en una situación emocionalmente relajada (cold) son incapaces de preveer la intensidad del sufrimiento que les puede producir la culpa o la vergüenza derivadas de violar una norma y los actos que pueden ser capaces de llevar a cabo en esas condiciones. A esto se refiere el cold to hot empathy gap. A la inversa, cuando los individuos se hallan en una situación emocionalmente intensa (hot) son incapaces de prever las consecuencias que pueden tener las acciones que llevan a cabo en esas condiciones. A esto se refiere el hot to cold empathy gap. El propio Elster (2007) ejemplifica estos fenómenos a partir de un caso real. En 1997 en Francia seis personas se suicidaron después de haber sido públicamente expuestas como consumidoras de material pedófilo. Resulta plausible suponer que fue la vergüenza derivada de ser descubierto violando una norma social la que los impulsó a quitarse la vida. Es igualmente probable suponer que si hubieran sido capaces de anticipar el sufrimiento que les generaría la vergüenza en caso de ser descubiertos, nunca habrían violado esa norma. A la inversa, si en el momento emocionalmente más intenso hubiesen sido capaces de prever que la emoción cesaría pronto, probablemente no se habrían suicidado.

\section{Conclusiones}

A lo largo de este trabajo se han tratado de poner en diálogo tres aproximaciones diferentes dentro de la tradición analítica a la cuestión de las normas: la de la TER-e, la de C. Bicchieri y la de Jon Elster.

Después de todo lo que ha venido argumentándose, cabe concluir que la distinción de Jon Elster es la que mejor captura la naturaleza de la conducta guiada por normas. Además, a consecuencia de ello, sus conceptos son los que pueden utilizarse de modo más fructífero en la explicación de los fenómenos sociales reales.

Jon Elster (2007) se ha venido mostrando crítico en los últimos tiempos con la utilización de modelos matemáticos formales en ciencias sociales así como escéptico con el potencial predictivo de estos (al menos en su estado de desarrollo actual). No obstante, no hay nada en los conceptos de Elster que impida su expresión en términos de función 
de utilidad y su posterior inclusión en un modelo matemático con vocación predictiva. Al contrario, el rigor en su definición y su informatividad los hacen especialmente adecuados para tales propósitos. Por otra parte, Elster utiliza habitualmente con éxito sus conceptos en el seno de modelos de mecanismos que explican causalmente los fenómenos sociales.

La clasificación de Jon Elster es muy similar a la de C. Bicchieri y, de modo particular, ambos tratan de capturar el mismo fenómeno cuando: uno, trazan sus distinciones entre normas cuasi-morales y sociales y, dos, entre normas sociales seguidas independientemente de la existencia de sanciones (2b) o como consecuencia de la existencia de sanciones (2b'). Según ha tratado de argumentarse en estas páginas, la clasificación del noruego captura de forma analíticamente más precisa en ese punto la naturaleza del fenómeno al que se dirige, se acerca más al ideal de la "clase natural", y ese es el elemento decisivo que decanta la balanza a favor de su clasificación.

\section{REFERENCIAS BibLIOGRÁFICAS}

Aguiar, F. y A. de Francisco. 2009. "Rational choice, social identity and beliefs about oneself". Philosophy of the Social Sciences 39: 547-571.

Bicchieri, C. 2006. The Grammar of Society. The Nature and Dynamics of Social Norms. Cambridge: Cambridge University Press.

Coleman, J. 1991. Foundations of Social Theory. Cambridge (MA): Harvard University Press.

Elster, J. 1991. El cemento de la sociedad: las paradojas del orden social. Barcelona: Gedisa.

Elster, J. 2002. Alquimias de la mente. La racionalidad y las emociones. Barcelona: Paidós/El Roure.

Elster, J. 2007. Explaining Social Behavior. More Nuts and Bolts for the Social Sciences. Cambridge: Cambridge University Press.

Elster, J. 2009a. "Social Norms and the Explanation of Behavior". Pp. 195-217 en The Oxford Handbook of Analytical Sociology, editado por P. Hedström y P. Bearman. New York: Oxford University Press.

Elster, J. 2009b. "Emotions". Pp. 51-71 en The Oxford Handbook of Analytical Sociology, editado por P. Hedström y P. Bearman. New York: Oxford University Press.

Linares, F. 2007. "El problema de la emergencia de normas sociales en la acción colectiva. Una aproximación analítica". Revista Internacional de Sociología 46:131-160.

Miller, D. T. y D. A. Prentice. 1994. "Collective errors and errors about the collective." Personality and Social Psychology Bulletin 20:541-550.

Sánchez-Cuenca, I. 2007. "Cooperar por principio." Revista Internacional de Sociología 46:11-35.

Ullmann-Margalit, E. 1977. The Emergence of Norms. Oxford: Clarendon Press. 
JoRd TENA-SÁNCHEZ es investigador postdoctoral en el Departamento de Sociología de la Universitat Autònoma de Barcelona y miembro del Grupo de Sociología Analítica y Diseño Institucional (GSADI). Su principal área de interés es la teoría social. En este ámbito investiga en el terreno de la sociología analítica, el diseño institucional y la filosofía política contemporánea.

ARIADNA GüelL-SanS es licenciada en Sociología por la Universitat Autònoma de Barcelona y Master en Gestión Pública por la Escola d'Administració Pública de Catalunya. Su principal área de interés es la política social y la gestión de políticas públicas. Actualmente trabaja en el Institut de Seguretat Pública de Catalunya en tareas de gestión de la formación.

RECIBIDO: $17 / 02 / 2009$

ACEPTADO: 03/03/2011 